\title{
Efficiency of a Three-Particle Energy Sink: Experimental Study and Numerical Simulation
}

\author{
S. S. Kevorkov, R. K. Khamidullin, I. P. Koroleva, \\ V. V.Smirnov, E. B. Gusarova, L. I. Manevich
}

The results of an experimental and numerical investigation of the dynamics of a string with three uniformly distributed discrete masses are presented. This system can be used as a resonant energy sink for protecting structural elements from the effects of undesirable dynamic loads over a wide frequency range. Preliminary studies of the nonlinear dynamics of the system under consideration showed its high energy capacity. In this paper, we present the results of an experimental study in which a shaker's table mounted cantilever beam was being protected. As a result, the efficiency of the sink was confirmed, and data were also obtained to refine the mathematical model. It was shown that the experimental data obtained are in good agreement with the results of computer simulation.

Keywords: nonlinear dynamics, nonlinear normal mode, limiting phase trajectory, energy exchange, localization

Received December 19, 2017

Accepted July 03, 2018

The authors are grateful to the Russian Foundation for Basic Research (grant 17-01-00582 A). This work was supported by the Program of Fundamental Researches of the Russian Academy of Sciences (project No. 0082-2014-0013, state registration number AAAA-A17-117042510268-5).

Sumbat S. Kevorkov

kevorkovss@gmail.com

Irina P. Koroleva

irakikotx@gmail.com

Valery V.Smirnov

vvs@polymer.chph.ras.ru

Elena B. Gusarova

elena.gusarova.530mail.ru

Leonid I. Manevich

manevitchleonid3@gmail.com

Semenov Institute of Chemical Physics Russian Academy of Science

ul. Kosygina 4, building 1, Moscow, 119991, Russia

Ruslan K. Khamidullin

brus5555550ya.ru

Bauman Moscow State Technical University

ul. 2-ya Baumanskaya 5, Moscow, 105005, Russia

RUSSIAN JOURNAL OF NONLINEAR DYNAMICS, 2018, 14(3), 355-366 


\section{Introduction}

The damping of mechanical vibrations, and the energy harvesting, are important tasks that represent a whole field of engineering science with a very wide range of applications - from industrial tasks to usage in everyday life. Some applications of this area include damping of vibrations in space and aero structures, protection of buildings from earthquakes and explosions, noise reduction in sound recording, vibration protection of various parts of machines and mechanisms, as well as passive accumulation of energy from the environment (for example, from mechanical vibrations of moving train parts or vibrations of tree branches from the wind), which is mainly used to power low-power wireless sensors.

Traditionally used vibration and shock absorbers provide oscillation suppression by using dampers or detuning from resonance [1]. Recently, an alternative approach to solving this problem has been proposed and justified, suggesting the use of resonant energy sinks [2-6]. The operating principle of such sinks in the condition of pulsed excitation is based on the resonance absorption (accumulation) of energy, transmitted by attached structure, followed by the going out of resonance due to a change in frequency depending on the amplitude of the oscillations. Therefore, the most important feature of an effective energy sink is that it belongs to a class of systems operating under conditions close to an acoustic vacuum. In the simplest case of a single-mass sink, this means the lack of natural frequency, which makes it possible to transfer energy in a wide frequency band.

Currently, sinks consisting of a single oscillator are being thoroughly studied and widely used in various fields of technology. Experimental studies of structure oscillations $[3,7,8]$ quenched by attaching a nonlinear oscillator to it showed that such a sink is quite efficient, in full agreement with the theoretical foundations given in $[2,3]$.

However, the question arises how to increase the efficiency and expand the range of operating frequencies of energy sinks that are capable of accumulating mechanical energy? Energy absorbers having a strongly nonlinear extended structure, such as strings with discretely distributed masses, may have a fundamental advantage over the low-dimensional analogs. Such sinks allow more resonances [9], which increases the range of their operating frequencies and efficiency. The increase in the number of masses in the oscillatory chain under consideration makes it possible to increase the number of achievable resonance regimes, which should result in an increase in the energy intensity and, consequently, in the efficiency of the energy sink. In this paper, we consider sinks consisting of three oscillators connected to each other and to the walls of a rigid frame by longitudinal and transverse strings without a preliminary stretching.

From a theoretical point of view, this system with an arbitrary number of masses was considered in $[9,10]$. It was shown that it allows a large number of resonances, and it was predicted that it can be used as an energy sink of increased efficiency. The present paper is devoted to an experimental investigation and verification of theoretical results.

\section{Experimental facility and its features}

The experimental facility is a chain of three oscillators with a mass of 30 grams each, connected together and with a frame by an elastic string (Fig. 1). According to the theoretical model of the sink, the chain in the equilibrium position should not be stretched. This ensured the absence of linear terms in the equations of motion, and, consequently, the functioning of the system in the acoustic vacuum regime. In the prototype, it is impossible to avoid string tension in the equilibrium position, however, in the experimental facility, the tension is not significant. 


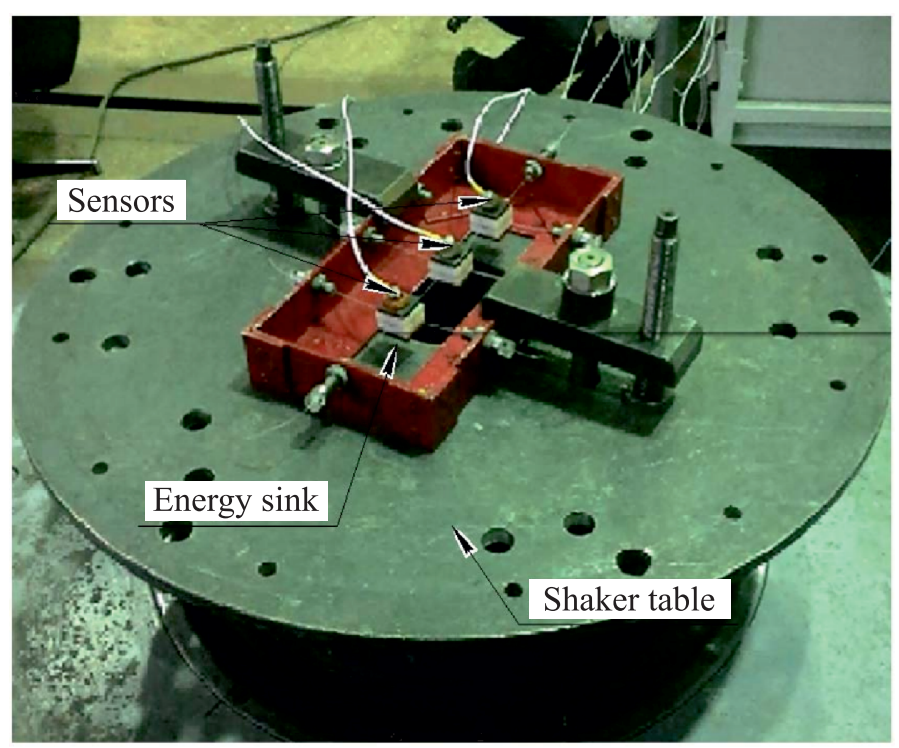

Fig. 1. Experimental setup.

It is difficult to verify this assumption directly, but we may judge about the significance of tension contribution from the results of vibration experiments and obtained amplitude-frequency relations. If the string tension was significant, then the sink would behave like a quasi-linear system, which is not the case.

The following problems have been set for research: determination of the optimal energy absorption frequency range and determination of the sink's efficiency as a device for protecting structures from shock loading.

To solve the first problem, the energy sink was fixed on the shaker's table, an acceleration sensor was mounted on each oscillator, and one more sensor fixed on the shaker's table was used to monitor the vibration regime. The vibration regime was selected to ensure acoustic vacuum conditions during the tests. At a large vibration amplitude, the strings stretched not elastically, which was fixed not only by the sensors, but also visually. It was found that the inelastic stretching of the string occurred at amplitudes greater than $10 \mathrm{~g}$. This phenomenon will be taken into account for designing the next prototype of the energy sink. It is planned to replace the strings with springs, which will significantly increase the loads allowable for the energy sink. The parameters of the final regimes of the resonance tests are given in Table 1 . To reveal the nonlinear dependence of the amplitude of resonant oscillations on their frequency, the tests were carried out at different loads, in direct regime (1.3) and reverse regime of scanning (2.4), Table 1.

Table 1. Experimental regimes

\begin{tabular}{|c|c|c|}
\hline Number of regime & Vibrational, $\mathrm{Hz}$ & Vibrational overload, $\mathrm{g}$ \\
\hline 1 & $10-60$ & 0.15 \\
\hline 2 & $60-10$ & 0.15 \\
\hline 3 & $10-60$ & 0.2 \\
\hline 4 & $60-10$ & 0.2 \\
\hline
\end{tabular}


The amplitude-frequency characteristics (AFC) obtained are shown in Figure 2. The resonant frequency of the system changed greatly depending on the direction of the scan, and insignificantly with a change in the load. Significant differences in the resonance frequencies $[12 \mathrm{~Hz}$ and $21 \mathrm{~Hz}]$ and amplitudes [5g and $9 \mathrm{~g}]$ obtained in the forward and reverse modes of scanning are consistent with the analytical solution and support the assumption that the system is strongly nonlinear and the test conditions are close to acoustic vacuum. For clarity, the AFC obtained in the analytical solution of the equations of motion in [11] are shown in Fig. 3.

The results obtained make it possible to consider the first problem as solved and allow one to determine the coefficient of damping and hence the effective coefficient of friction.
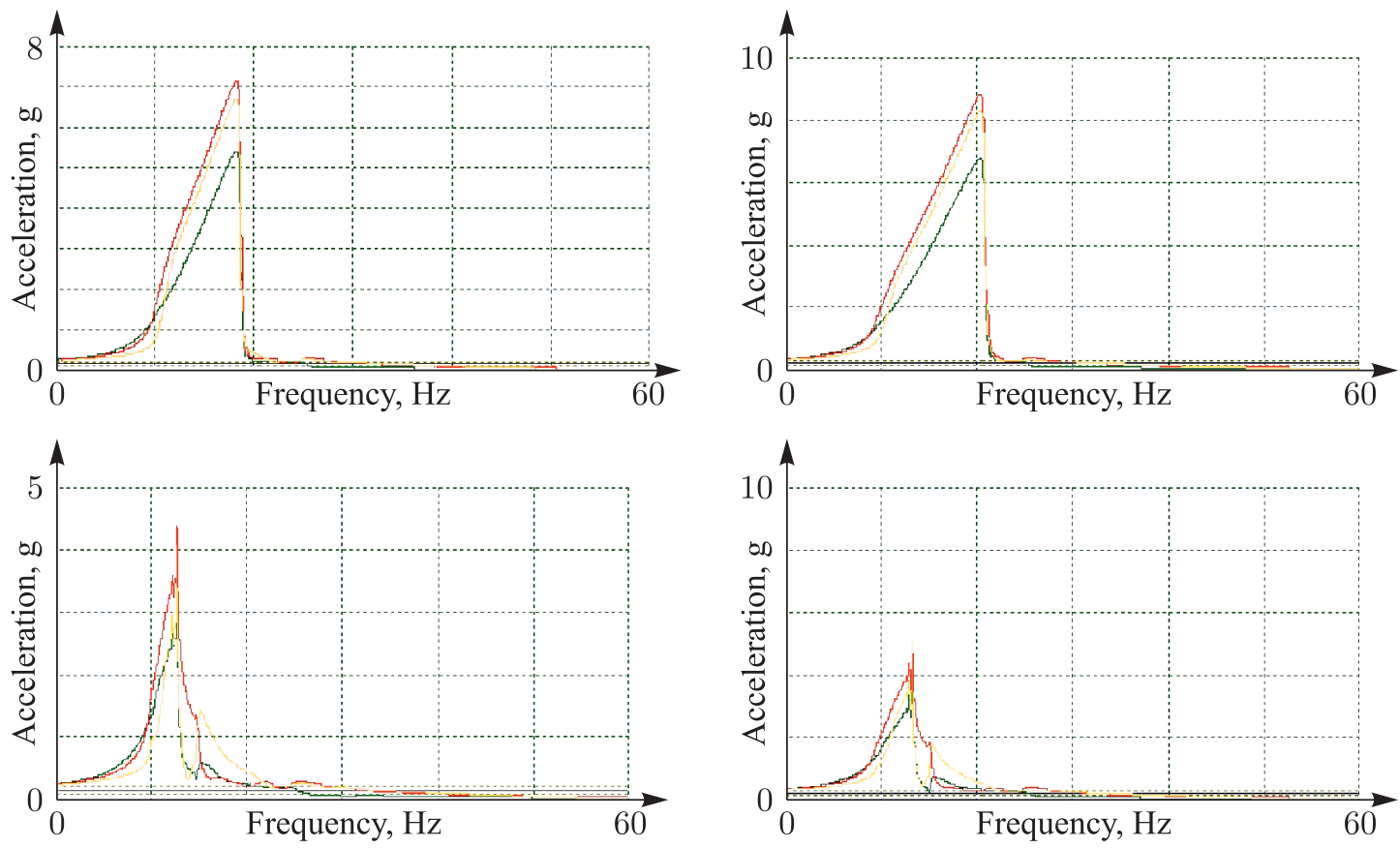

Fig. 2. Amplitude-frequency characteristics. Upper plot: AFC of the energy sink under frequency change from 10 to $60 \mathrm{~Hz}$ (left) and in inverse direction (right); load was $0.15 \mathrm{~g}$. Lower plot-the same for load $0.2 \mathrm{~g}$.

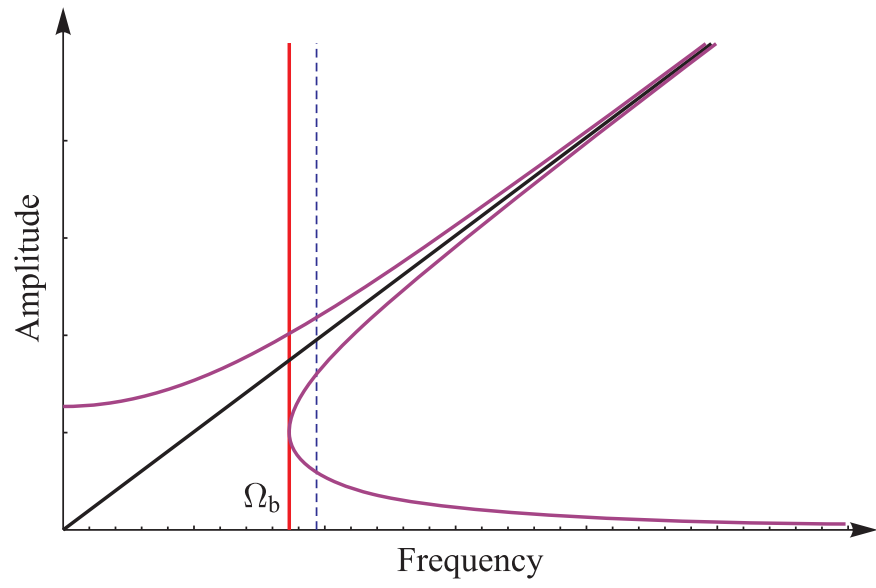

Fig. 3. Teoretical AFC of energy sink. 


\section{The efficiency of the energy sink}

As noted above, the shaker's table mounted cantilever beam, with prototype's frame fixed on its free end (Fig. 4) was used to determine the efficiency of the energy sink. The arrangement of the sensors is shown in Figure 4b. The beam shape ensures that the intrinsic frequency of the protected structure falls within the effective absorption frequency range of the energy sink. The weight of the beam was $1.51 \mathrm{~kg}$, the weight of the energy sink frame was $0.657 \mathrm{~kg}$, the total mass of the oscillators and the strings was $0.095 \mathrm{~kg}$.

The construction was loaded with a vibroshock, the characteristic of which is given in Table 2. The spectral and time characteristics of oscillations of the monitoring sensor in the case of a vibroshock are shown in Fig. 5. Vibroshock is often used in impact and seismic tests, simulated explosions, etc. The choice of such an impact is due to the visibility of the results obtained and the ability to provide an effective short time load with a fast achievement of the maximum of amplitude.

Identical vibroshock loads were applied to the construction with a nonfunctioning and functioning energy sink. The time records of damped oscillations for both loading cases are shown in Fig. 6. The green color indicates the oscillations of sensor 1 (the sensor with the largest amplitude), blue stands for the oscillations of sensor 2 (the monitoring sensor). The comparison results of the construction's behavior with functioning and nonfunctioning energy sink are given in Table 3. The dissipation time is the time of decrease in the oscillations amplitude to $0.1 \mathrm{~g}$. Thus, the efficiency of the energy sink is evidenced by a decrease in the dissipation time by a factor of 2.52 and a maximum of amplitude by a factor of 1.25 .
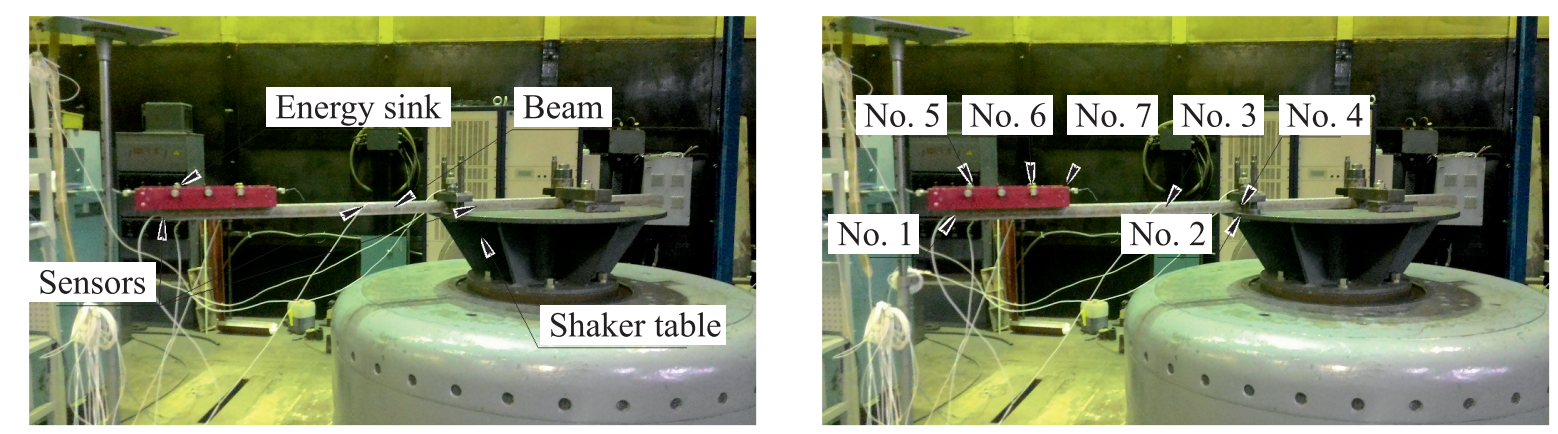

Fig. 4. Experimental facility. Sensors 1, 3 and 4 are on the beam, sensor 2 is driving, sensors 5 and 6 are on the masses of the energy sink, and sensor 7 is on the frame.

Table 2. Parameters of vibrational impact

\begin{tabular}{|c|c|c|}
\hline Frequency range, $\mathrm{Hz}$ & Load, $\mathrm{g}$ & Time of impulse, $\mathrm{s}$ \\
\hline $10-50$ & 0.8 & 0.7 \\
\hline
\end{tabular}

Table 3. Efficiency of the energy sink

\begin{tabular}{|l|c|c|}
\hline Parameter & Without the sink & With the sink \\
\hline Dissipation time, s & 8.88 & 3.53 \\
\hline Maximal amplitude of overloading, $\mathrm{g}$ & 6.68 & 5.32 \\
\hline
\end{tabular}



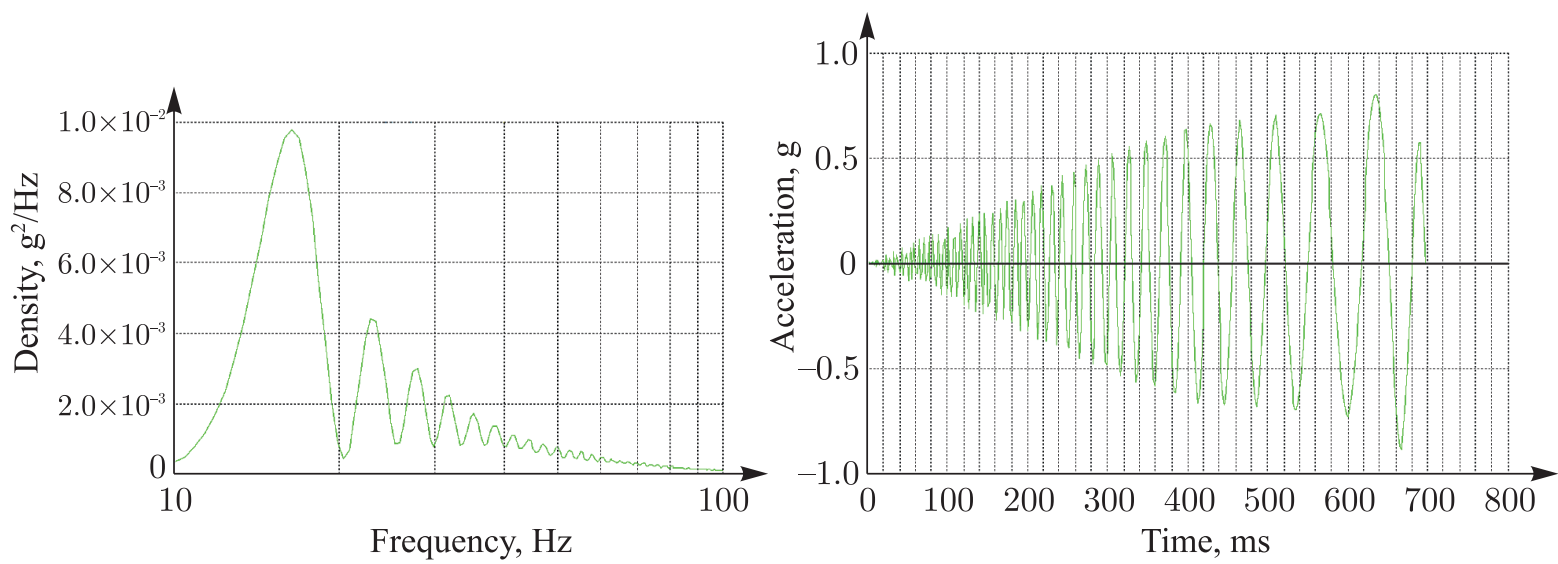

Fig. 5. Spectrum of the driving sensor during the vibrational impact and time evolution of driving amplitude.

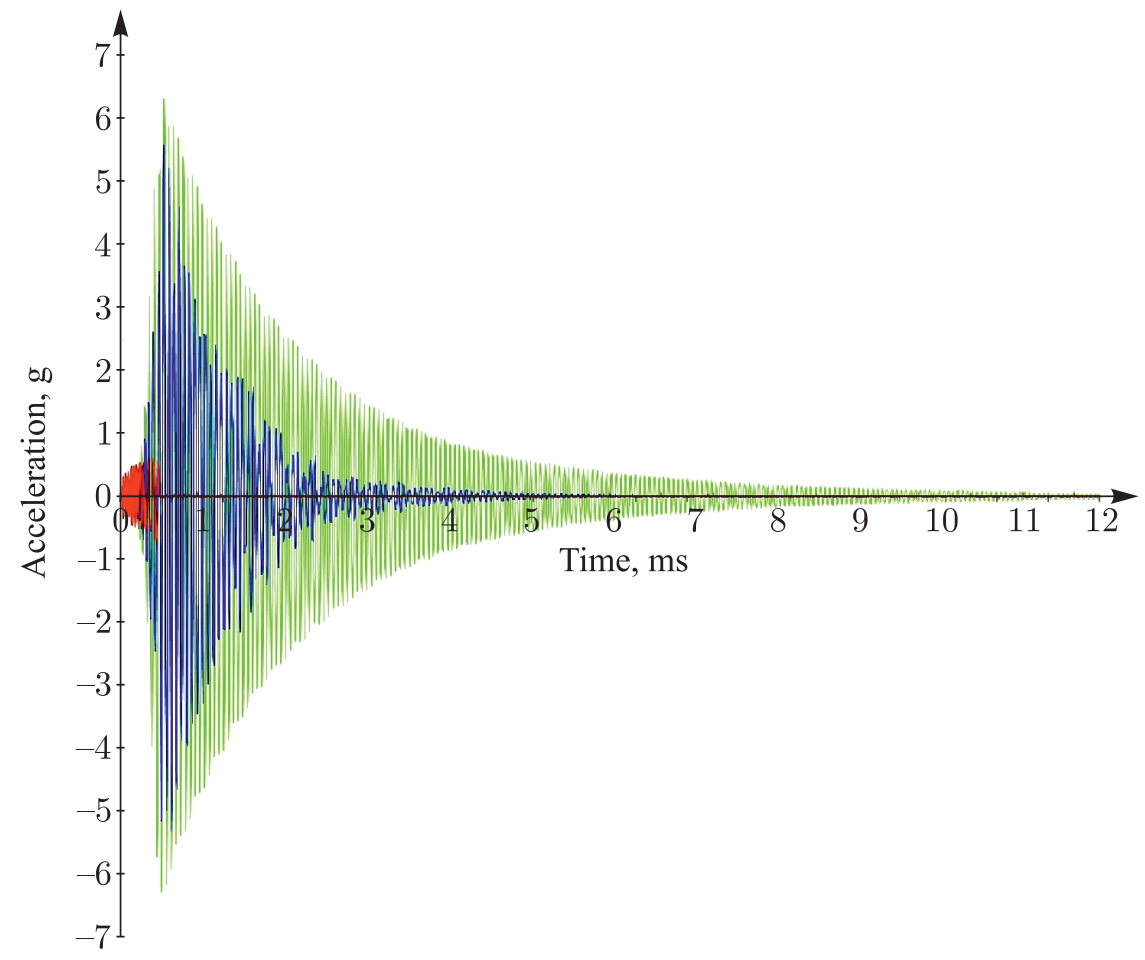

Fig. 6. Time evolution of amplitude with the sink (blue) and without it (green). The red line depicts the amplitude of impact (the amplitude of the vibroshaker).

\section{The energy sink model and the equations of motion in the conservative limit}

The article's final part is devoted to numerical modeling of the equations of motion of the system under consideration. First of all, we show that the basic regime that ensures the effective transfer of energy to the sink can be determined already in the conservative limit. 


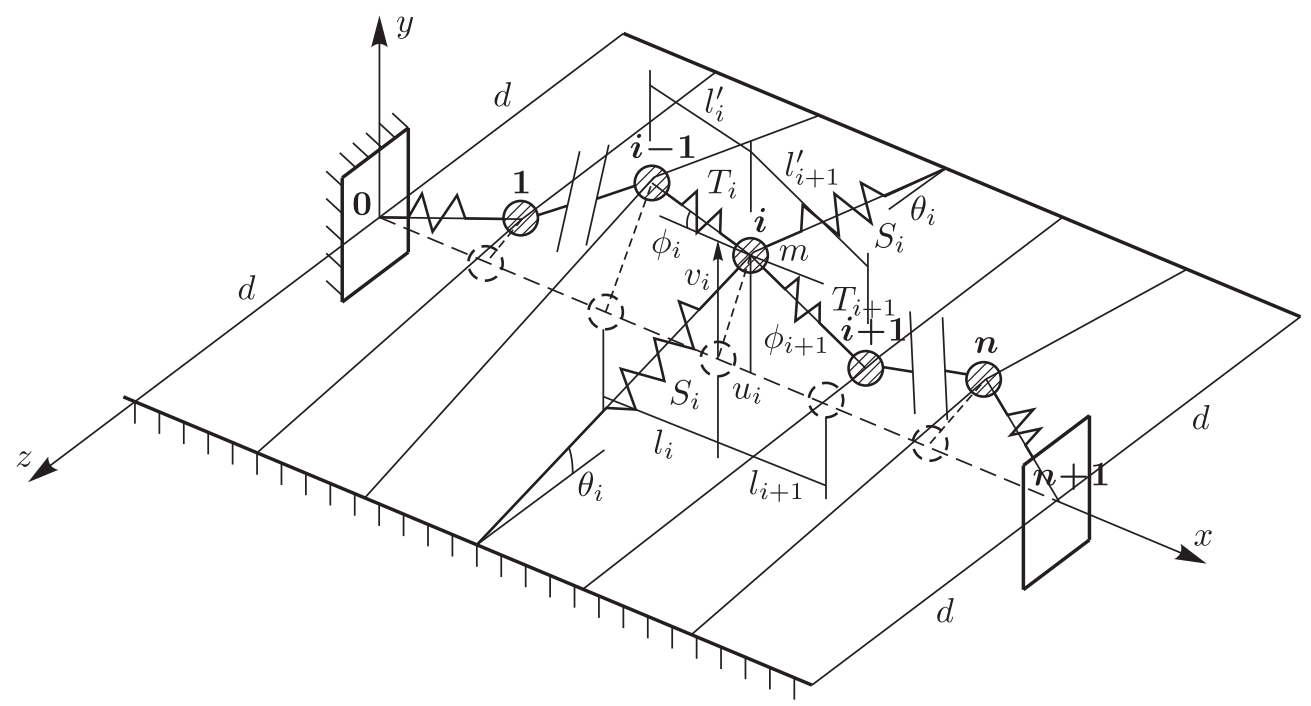

Fig. 7. Oscillatory chain with elastic support. Only one transversal string is shown in order to make the picture more readable.

Consider a prestretched string with uniformly distributed and discrete masses equal in magnitude (see Fig. 7) experiencing the action of restoring forces proportional to the cube of displacement with the coefficient $c$. The equations of motion of such a system read as follows:

$$
\begin{array}{ll}
m \ddot{U}_{j}+2 F_{j U}+T_{j} \cos \varphi_{j}-T_{j+1} \cos \varphi_{j+1}=0, & j=1, \ldots, N \\
m \ddot{V}_{j}+2 F_{j V}+T_{j} \sin \varphi_{j}-T_{j+1} \sin \varphi_{j+1}=0, & j=1, \ldots, N
\end{array}
$$

where $U_{j}, V_{j}$ are the longitudinal and transverse displacements of the $j$ th mass, respectively, $\varphi_{j}$ is the angle of rotation of the $j$ th segment relative to its position in the undeformed string, $T_{j}$ are the tension forces of longitudinal strings, $F_{j}$ is the tension forces of transverse strings $\left(F_{j U}, F_{j V}\right.$ is the projection of this force on the vertical and longitudinal axes). For simplicity, it is assumed that there is no motion along the third axis. From symmetry considerations, the tension forces of transverse strings are the same, hence the coefficient is 2 . The tension forces of individual segments of a longitudinal string are proportional to their deformations and depend on the displacements as follows:

$$
T_{j}=K_{1}\left[\left(U_{j}-U_{j-1}\right)+\frac{1}{2 l}\left(V_{j}-V_{j-1}\right)^{2}\right],
$$

where $l$ is the length of the undeformed segment of the string and $K_{1}$ is the stiffness coefficient of the longitudinal strings. The tension forces of transverse strings are equal. $F_{j U}=$ $=K_{2} \frac{\left(U_{j}^{2}+V_{j}^{2}\right) U_{j}}{2 d^{2}}, F_{j, V}=K_{2} \frac{\left(U_{j}^{2}+V_{j}^{2}\right) V_{j}}{2 d^{2}} . K_{2}$ is the stiffness coefficient of transverse strings.

As noted above, in the absence of a substrate, the nonlinear normal modes (NNM) of the system under consideration with rectilinear trajectories coincide with the modes of the linear oscillatory chain [12]. Each mode (with the number $m$ ) is described by the law of motion: $V_{j}^{m}(t)=a_{m}(t) \sin \frac{\pi j m}{N+1}$, with a frequency that depends on the amplitude and is proportional to the square of the corresponding oscillations frequency of the linear oscillatory chain with 
the same wave number:

$$
\omega_{m}^{2}=4 \sin ^{2} \frac{\pi m}{2(N+1)} .
$$

With the introduction of transverse strings, the situation becomes more complicated. NNM of the string cease to be exact solutions. However, they are now approximate NNM. In [9], a condition for the existence of a resonance between modes is derived:

$$
\frac{\frac{3}{\mu}+3 \omega_{m}^{4}-\omega_{m}^{2} \omega_{k}^{2}}{\frac{3}{\mu}+3 \omega_{m}^{4}-\omega_{m}^{2} \omega_{k}^{2}}>0 .
$$

For three oscillators this means that the 1 st and 2 nd modes resonate at $\mu<21.43$, the 2 nd and the 3rd at any $\mu$, and the 1st and the 3rd at $\mu<3.09$. Thus, for all values of $\mu<3.09$, all three modes resonate. This indicates the greatest efficiency of the sink at this ratio of parameters, because the energy will be transferred from the protected structure to all modes at once, which should eventually lead to more intense dissipation (in the presence of friction). In our case, $\mu=5.6184$.

\section{Equations of motion of the system under consideration in the conservative limit}

For simplicity, we restrict ourselves to considering beam oscillations corresponding to its first normal mode. Then the mathematical description of the dynamic process in the beam can be reduced to the linear oscillator model:

$$
M_{\text {effect }} \ddot{X}(t)+K_{\text {effect }} X(t)=0,
$$

where $X(t)$ is the displacement of the unfixed end of the beam. The effective rigidity can be estimated from the beam equation; it is known that if a static force $F$ is applied to the end of a cantilever beam, then the displacement of the free end of the beam will be: $\Delta x=F \frac{L^{3}}{3 E J}$. Then the effective rigidity is $K_{\text {effect }}=\frac{3 E J}{L^{3}}$, where $E$ is the elasticity modulus of the material (aluminum), $J$ the beam's moment of inertia, $L$ the length of the loose part of the beam. The oscillation frequency of the beam was determined experimentally: $\omega=17 G z=17 \cdot 2 \pi s^{-1}$. Knowing the frequency and effective rigidity, the effective mass can be found: $M_{\text {effect }}=K_{\text {effect }} / \omega^{2}$. The numerical values are given in Table 4 . With a functioning energy sink, we integrate such a system (where $V_{j}$ is the vertical displacement of the oscillation of the sink relative to the frame or the beam, which is the same):

$$
\begin{array}{ll}
m \ddot{U}_{j}+T_{j} \cos \theta_{j}-T_{j+1} \cos \theta_{j+1}=0, & j=1, \ldots, N \\
m \ddot{V}_{j}+2 F_{j}+T_{j} \sin \theta_{j}-T_{j+1} \sin \theta_{j+1}=-m \ddot{X}(t), & j=1, \ldots, N \\
\ddot{X}(t)+\omega^{2} X(t)=\left(1 / M_{\text {effect }}\right)\left(T_{1} \sin \theta_{1}-T_{N+1} \sin \theta_{N+1}\right) . &
\end{array}
$$

We consider this system under initial conditions corresponding to the initial momentum: $X(0)=0, \dot{X}(0)=v_{0}$. At the initial instant of time, the sink oscillators are at rest. Since the equations of motion were written in relative coordinates, their relative velocity will be equal 

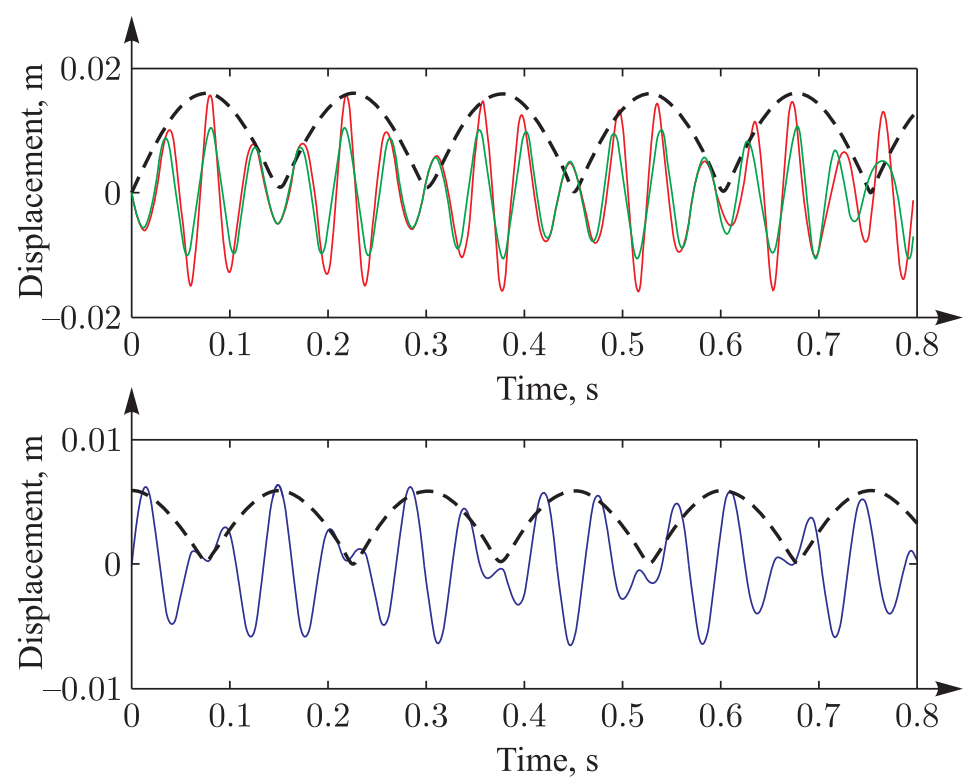

Fig. 8. Beating regime. The upper plot shows oscillations of the central (red) mass and outside (green) masses. The lower one shows the oscillation of the beam end.

to the speed of the beam with the opposite sign: $\dot{V}_{1}(0)=\dot{V}_{2}(0)=\dot{V}_{3}(0)=-v_{0} . V_{1}(0)=V_{2}(0)=$ $=V_{3}(0)=0$. For a certain value of $v_{0}$, the beat condition is observed, which is possible when the oscillation frequencies of the sink and the beam are close. For the practical use of the sink, this regime is fundamentally important. This regime is fundamentally important for the practical use of the sink. In the conservative system, energy is transferred not only from the beam to the sink, but also back. Figure 8 shows the beating regime, which is observed at the initial velocity $=0.7 \mathrm{~m} / \mathrm{s}$.

However, in the presence of friction, the situation drastically changes: the energy is transferred to the sink, where it is partially dissipated. In this case, the beam oscillations amplitude and its dissipation time decrease. Due to the fact that the sink functions in the regime close to an acoustic vacuum, the frequency of oscillations, which depends directly on the amplitude, decreases with dissipation. That is the reason why the reverse outflow of energy is much less.

\section{Accounting for the dissipation influences}

Now consider the real sink, taking into account the friction in the system (friction is taken into account effectively). For a functioning and nonfunctioning sink, the logarithmic damping decrements were calculated separately. Their numerical values are given in Table 4 . The external force is taken into account in the form of readings of sensors mounted on the sink, which are used as the right-hand part (i.e., the sink is a noninertial system with a given acceleration).

Thus, to simulate the complete system, the following system of equations of motion with zero initial conditions was integrated:

$$
\begin{aligned}
& m \ddot{U}_{j}+m \nu \dot{U}_{j}+T_{j} \cos \theta_{j}-T_{j+1} \cos \theta_{j+1}=0, \quad j=1, \ldots, N \\
& m \ddot{V}_{j}+m \nu \dot{V}_{j}+2 F_{j}+T_{j} \sin \theta_{j}-T_{j+1} \sin \theta_{j+1}=-m \ddot{X}(t)-m a_{v i b r}(t), \quad j=1, \ldots, N \\
& \ddot{X}(t)+\nu_{s h v} \dot{X}(t)+\omega^{2} X(t)=-a_{v i b r}(t)+\left(1 / M_{\text {effect }}\right)\left(T_{1} \sin \theta_{1}-T_{N+1} \sin \theta_{N+1}\right) .
\end{aligned}
$$


There are some distinctions in the real experimental facility from its model, caused by technical reasons. As one of such distinctions, we can specify an asymmetric string response. In Section 5, it was assumed that the strings connecting the oscillators work both in compression and in tension. For technical reasons, in a real system, strings work only on stretching. In the numerical calculation, this was not taken into account. However, it can be noted that taking the asymmetry into account hardly affects the result of simulation. In addition, gravity is not taken into account in our model. However, its influence is small, because the mass of the sink oscillators is relatively small.

It should be noted that so far only the single-oscillator sink has been experimentally investigated and applied. A natural question arises: how does the number of oscillators affect the efficiency of the sink. Our studies of the nonlinear dynamics of the sink $[9,13]$ suggested that the efficiency of the sink would increase with the increase in the number of oscillators due to the appearance of multiple resonances. Therefore, after performing the first experimental study with a three-mass sink and making sure that the experimental results are in good agreement with the data of numerical simulation, we were able to compare the effects achieved with the use of a single-oscillator and three-oscillator sinks. Comparison of Fig. 9 and 10 shows that the transition to a three-oscillator sink really improves the efficiency of vibration suppression in the future, both experimental and theoretical studies are planned using energy sinks containing a different number of masses.

Table 4. The numerical values of the parameters used in the calculations

\begin{tabular}{l|c|c|c} 
Parameter & Symbol & Value & Dimension \\
\hline Stiffness of longitudinal springs & $K_{1}$ & 18726 & $\mathrm{~N} / \mathrm{m}$ \\
Stiffness of transverse springs & $K_{2}$ & 3333 & $\mathrm{~N} / \mathrm{m}$ \\
Elasticity modulus of the beam & $E$ & 70 & $\mathrm{GPa}$ \\
Inertia moment of the beam & $J$ & $2.7 \cdot 10^{-8}$ & $\mathrm{~m}^{4}$ \\
Logarithmic decrement of the oscillators & $\nu / 2$ & 0.4 & $\mathrm{~s}^{-1}$ \\
Logarithmic decrement of beam & $\nu_{s h v} / 2$ & 0.54 & $\mathrm{~s}^{-1}$ \\
Oscillator mass & $\mathrm{m}$ & 0.03 & $\mathrm{~kg}$
\end{tabular}

\section{Conclusions}

For the first time, experimental results have been obtained that attest to the effectiveness of a three-oscillator energy sink under conditions of intense short-term vibroshock action. It is shown that its application leads to a substantial decrease in the amplitude of the oscillations and decay time. A "basic" regime of complete energy exchange between the beam structure and the energy sink is singled out in the limiting case of a conservative system, the transformation of which, taking dissipation into account, ensures the high efficiency of the tested sink. A fundamentally important conclusion for further research is that the transition from a single-oscillator to a three-oscillator sink leads to a significant increase in efficiency. This means that the study of the dependence of energy absorption on the number of oscillators is very promising. 


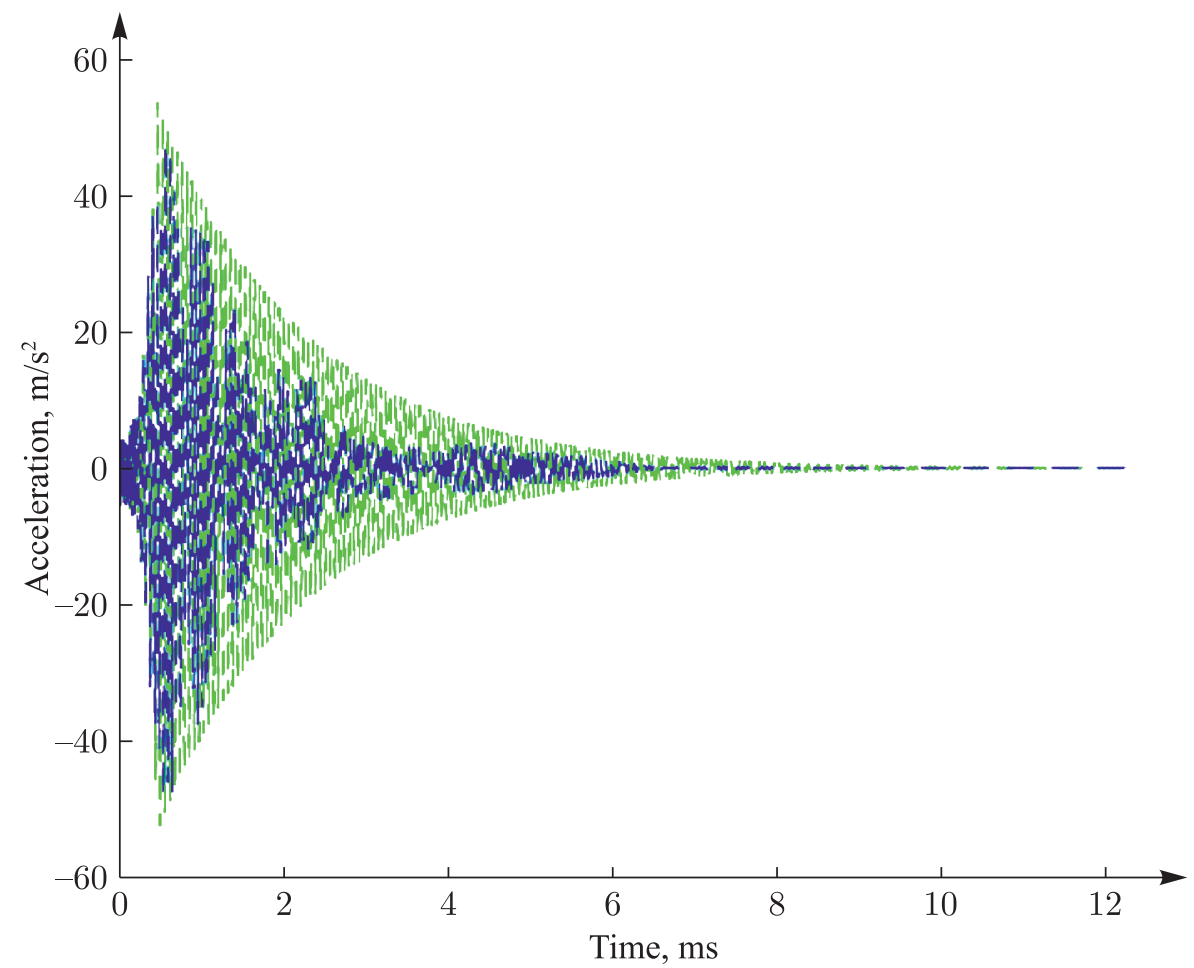

Fig. 9. Comparison of beam dynamics without and with the three-oscillator sink (results of numerical simulation).

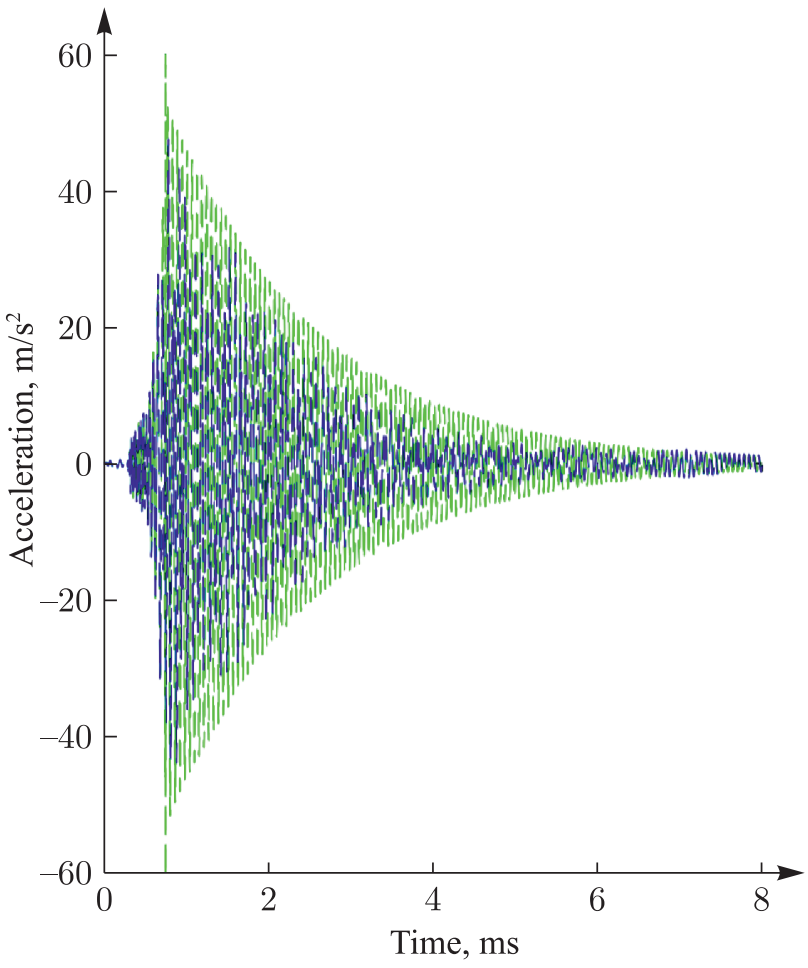

Fig. 10. Comparison of beam dynamics without and with the one-oscillator sink (results of numerical simulation). 


\section{References}

[1] Den Hartog, J.P., Mechanical Vibrations, 4th ed., New York: McGraw-Hill Book Company, 1956.

[2] Gendelman, O. V., Transition of Energy to a Nonlinear Localized Mode in a Highly Asymmetric System of Two Oscillators, Nonlinear Dyn., 2001, vol. 25, nos. 1-3, pp. 237-253.

[3] Manevitch, L. I., Gourdon, E., and Lamarque, C.-H., Towards the Design of an Optimal Energetic Sink in a Strongly Inhomogeneous Two-Degree-of-Freedom System, J. Appl. Mech., 2007, vol. 74, no. 6, pp. 1078-1086.

[4] Gendelman, O. V., Manevitch, L. I., Vakakis, A. F., and M'Closkey, R., Energy Pumping in Nonlinear Mechanical Oscillators: Part 1. Dynamics of the Underlying Hamiltonian Systems, J. Appl. Mech., 2001, vol. 68, no. 1, pp. 34-41.

[5] Vakakis, A. F., Gendelman, O. V., Bergman, L. A., McFarland, D. M., Kerschen, G., and Lee, Y.S., Nonlinear Targeted Energy Transfer in Mechanical and Structural Systems, Dordrecht: Springer, 2009.

[6] Manevitch, L.I., Kovaleva, A., Smirnov, V., and Starosvetsky, Yu., Quasi-One-Dimensional Nonlinear Lattices, in Nonstationary Resonant Dynamics of Oscillatory Chains and Nanostructures, Singapore: Springer, 2018, pp. 85-140.

[7] Gourdon, E., Lamarque, C.-H., and Pernot, S., Contribution to Efficiency of Irreversible Passive Energy Pumping with a Strong Nonlinear Attachment, Nonlinear Dyn., 2007, vol. 50, no. 4, pp. 793-808.

[8] Jiang, X., McFarland, D. M., Bergman, L.A., and Vakakis, A. F., Steady State Passive Nonlinear Energy Pumping in Coupled Oscillators: Theoretical and Experimental Results, Nonlinear Dyn., 2003, vol. 33, no. 1, pp. 87-102.

[9] Koroleva (Kikot), I. P. and Manevitch, L. I., Oscillatory Chain with Grounding Support in Conditions of Acoustic Vacuum, Nelin. Dinam., 2015, vol.11, no.3, pp.487-502 (Russian).

[10] Manevitch, L. I. and Koroleva, I. P., Limiting Phase Trajectories As an Alternative to Nonlinear Normal Modes, Proc. IUTAM, 2016, vol. 19, pp. 144-151.

[11] Smirnov, V.V. and Manevitch, L. I., Forced Oscillations of the String under Conditions of Sonic Vacuum, Philos. Trans. Royal Soc. A, 2018 (in press).

[12] Manevitch, L. I. and Vakakis, A. F., Nonlinear Oscillatory Acoustic Vacuum, SIAM J. Appl. Math., 2014, vol. 74, no. 6, pp. 1742-1762.

[13] Koroleva (Kikot), I. P., Manevitch, L.I., and Vakakis, A. F., Non-Stationary Resonance Dynamics of a Nonlinear Sonic Vacuum with Grounding Supports, J. Sound Vibration, 2015, vol.357, pp. 349-364. 